\title{
Biologia populacional das espécies de Ampithoidae (Crustacea, Amphipoda) associadas a Sargassum filipendula (Phaeophyta, Fucales) na Praia da Fortaleza, Ubatuba, São Paulo, Brasil
}

\author{
Giuliano B. Jacobucci ${ }^{1} \&$ Fosca P. P. Leite ${ }^{2}$ \\ ${ }^{1}$ Instituto de Biologia, Universidade Federal de Uberlândia. 38402-400 Uberlândia, Minas Gerais, Brasil. \\ E-mail: jacobucci@inbio.ufu.br \\ ${ }^{2}$ Departamento de Zoologia, Instituto de Biologia, Universidade Estadual de Campinas. 13083-970 Campinas, São Paulo, Brasil.
}

\begin{abstract}
Population biology of Ampithoidae species (Crustacea, Amphipoda) associated with Sargassum filipendula (Phaeophyta, Fucales), at Fortaleza beach, Ubatuba, São Paulo, Brazil. The population biology of three species of the family Ampithoidae (Ampithoe ramondi, Cymadusa filosa e Sunampithoe pelagica) associated with Sargassum filipendula at Fortaleza beach, northern coast of São Paulo state was investigated from June 2000 to May 2001. The amphipods were identified, counted and separated in size classes through a nested sieve set. Month hystograms were obtained for juveniles, males and females. The ampithoid sex ratio was determined monthly. The reproductive biology of the species was evaluated by the relative frequency of ovigerous females, fecundity, average egg volume and correlation analysis between head lengh of ovigerous females and number of eggs in its brood pouch. Ampithoe ramondi was the most abundant species. The higher densities of the three species occurred in spring and summer. The sex ratio presented a significant difference from 1:1 only for $A$. ramondi (1:1.2; $\chi^{2}=4.37$; g.l. $\left.=1 ; \mathrm{p}<0.05\right)$. Ovigerous females were registered for almost all the sampling periods. A positive correlation between the female head lengh and the number of eggs were only registered for $A$. ramondi $\left(r^{2}=0.27\right.$; $\mathrm{p}<0.05 ; \mathrm{n}=33$ ). Cymadusa filosa showed the greatest fecundity and egg volume.
\end{abstract}

KEY WORDS. Amphipods; macrofauna; phytal; population dynamics.

RESUMO. A biologia populacional de três espécies de anfípodes da família Ampithoidae (Ampithoe ramondi, Cymadusa filosa e Sunampithoe pelagica) associadas a Sargassum filipendula da Praia da Fortaleza, litoral norte do Estado de São Paulo foi estudada entre junho de 2000 e maio de 2001. Os anfípodes foram identificados, contados e separados em classes de tamanho através de um conjunto de peneiras agrupadas. Foram obtidos histogramas mensais de jovens, machos e fêmeas. A razão sexual dos ampitoídeos foi avaliada mensalmente. A biologia reprodutiva das espécies foi avaliada através freqüência relativa mensal de fêmeas ovígeras, de sua fecundidade, volume médio dos ovos e correlação do tamanho da cabeça com o número de ovos contidos em seus marsúpios. Ampithoe ramondi foi a espécie mais abundante. As maiores densidades para as três espécies ocorreram na-primavera e no verão. A razão sexual apresentou diferença significativa de $1: 1$ apenas para $A$. ramondi $\left(1: 1,2 ; \chi^{2}=4,37 ;\right.$ g.l. $=1 ; \mathrm{p}<0,05)$. Fêmeas ovígeras foram registradas em quase todos os períodos de amostragem. Correlação positiva baixa entre o tamanho da cabeça da fêmea e o número de ovos contidos na bolsa incubadora foi registrada somente para $A$. ramondi $\left(\mathrm{r}^{2}=0,27 ; \mathrm{p}<0,05 ; \mathrm{n}=33\right)$. Cymadusa filosa apresentou a maior fecundidade e o maior volume dos ovos.

PALAVRAS-CHAVE. Anfípodes; dinâmica populacional; fital; macrofauna.

O ambiente fital é dominado por macrófitas bentônicas representadas predominantemente por algas talosas e gramas marinhas (Edgar \& Moore 1986). Dentre os vários grupos de animais que vivem em associação com as macrófitas, os anfípodes constituem um dos taxa mais representativos em riqueza de espécies e abundância de organismos (EdGAR 1983a, Norton \& Benson 1983, Jacobucci \& Leite 2002).
Na costa sudeste do Brasil bancos de algas pardas do gênero Sargassum C. Agardh, 1820 são particularmente conspícuos em costões de áreas rasas podendo representar mais de $80 \%$ da cobertura e biomassa de algas em determinadas áreas do litoral dos Estados de São Paulo e Rio de Janeiro (Paula \& OLIveira Filho 1980, Széchy \& Paula 2000).

Muitas espécies de anfípodes ocorrem associadas aos ban- 
cos de Sargassum do litoral paulista (TARARAM \& WAKABARA 1981, WAKABARA et al. 1983), incluindo-se representantes da família Ampithoidae, Ampithoe ramondi Audouin, 1826, Cymadusa filosa Savigny, 1816 e Sunampithoe pelagica H. Milne Edwards, 1830. A presença destas espécies tubícolas (BARnARD \& Karaman 1991a, b) ocorrendo em simpatria, já foi verificada em bancos de Sargassum de várias áreas do litoral norte do Estado de São Paulo (TARARAM \& W AKabara 1981, Leite et al. 2000, TAnaka \& Leite 2004). O sucesso dessas três espécies no mesmo ambiente pode estar relacionado a diferentes aspectos de sua biologia, incluindo diferenças nos períodos reprodutivos, na fecundidade e na duração do desenvolvimento embrionário (Steele \& Steele 1975, Leite 1996a).

Tomando-se por base trabalhos desenvolvidos em regiões temperadas envolvendo a predação da macrofauna associada a macrófitas (HoLmLund et al. 1990, SALA 1997) ea herbivoria de macroalgas por anfípodes (Duffy 1990), o potencial dos ampitoídeos, como recurso alimentar para peixes e elemento estruturador dos bancos de Sargassum da costa sudeste parece ser bastante promissor.

Embora existam trabalhos abordando aspectos da morfologia e do ciclo de vida dos ampitoídeos (Leite et al. 1986, LeITE 1996b, C, LeITE \& GüTH 2003), dados relativos à dinâmica populacional e reprodução de Ampithoe ramondi, Cymadusa filosa e Sunampithoe pelagica associados a Sargassum ainda são restritos (LEITE 1996b).

Estudos que permitam aprofundar o conhecimento da biologia das espécies de ampitoídeos são fundamentais para a compreensão do papel desses anfípodes em sistemas complexos como os bancos de Sargassum. Nesse sentido, este trabal ho buscou o conhecimento da biologia populacional das três espécies de Ampithoidae presentes em um banco de Sargassum filipendula C. Agardh, 1824 do litoral norte do Estado de São Paulo. Mais especificamente, procurou-se descrever a variação da estrutura populacional das espécies ao longo do ano e caracterizar al guns aspectos de sua atividade reprodutiva.

\section{MATERIAL E MÉTODOS}

\section{Área de estudo}

As coletas foram realizadas na região de infralitoral da Praia da Fortal eza ( $23^{\circ} 32^{\prime} \mathrm{S}, 45^{\circ} 10^{\prime} \mathrm{W}$ ) localizada na enseada da Fortaleza, município de Ubatuba, litoral norte do Estado de São Paulo. Este local é formado por uma feição rochosa estreita que avança na direção sudoeste-nordeste, apresentando dois lados com diferentes graus de exposição ao batimento das ondas (observação pessoal).

O costão onde foram realizadas as coletas apresenta cerca de 1,5 m de profundidade, considerando-se o nível da maré baixa, sendo moderadamente protegido da ação das ondas (SZÉCHY \& PAULA 2000). Embora Sargassum filipendula seja a alga dominante no local (observação pessoal), Dictyopteris delicatula J. V. Lamouroux, 1809, Dictyota cervicornis Küetzing, 1859 e Hypnea musciformis (Wulfen in Jacquin) J. V. Lamouroux, 1813 também são bastante conspícuas, e são encontradas na forma de epífitas sobre Sargassum (Paula \& Oliveira Filho 1980, Széchy $\&$ PAULA 2000).

\section{Coleta e tratamento das amostras}

As amostras de Sargassum filipendula foram coletadas em uma área previamente delimitada, no infralitoral raso, em um setor com $50 \mathrm{~m}$ de extensão. Este setor foi dividido em intervalos de 0,5 m totalizando 100 intervalos. Mensalmente entre junho de 2000 e maio de 2001 foram sorteados 15 intervalos do setor e em cada intervalo foi coletada uma fronde de Sargassum filipendula. As frondes foram raspadas do substrato com auxílio de uma espátula e envolvidas em sacos plásticos, aos quais foi adicionada posteriormente solução a 4\% deformal deído em água do mar. A amostragem foi realizada através de mergulho livre. A temperatura superficial da água foi obtida ao término de cada coleta em três pontos do setor de amostragem, obtendo-se um valor médio mensal de temperatura.

No laboratório, cada fron de foi lavada separadamente em um cristalizador e o material resultante foi filtrado em rede de 0,2 mm. Segundo TANAKA \& LeITE (1998), malhas com esse diâmetro de poro retêm mais de $99 \%$ dos anfípodes. As epífitas aderidas às frondes de Sargassum filipendula foram removidas manualmente. Para a obtenção do peso seco, cada fronde de S. filipendula foi colocada em estufa a 60 으 por 48 horas e pesada, snedo o mesmo procedimento realizado para as epífitas.

Os animais foram fixados em álcool $70 \%$ para identificação e contagem. Os Ampithoidae foram triados e identificados após a separação do total de animais associados à alga e, em seguida, passados numa série de telas de nylon de malha decrescente $(8,4,2,1,4,1,0,5$ e 0,2 mm) de modo a se obter uma separação dos anfípodes em diferentes classes detamanho (adaptado de EDGAR 1990). Para a identificação das espécies foram utilizados Bousfield (1973), Ruffo (1982), Myers (1985) e Barnard \& Karaman (1991a, b). A densidade das espécies de anfípodes associados a cada fronde de $\mathrm{S}$. filipendula foi obtida dividindo-se o número total de indivíduos pelo peso seco da alga em gramas.

A checagem do sexo dos indivíduos foi baseada na presença de oostegitos, sendo estas estruturas evidenciadas em fêmeas a partir da classe de 1,4 $\mathrm{mm}$. Todos os anfípodes desta classe de tamanho e das superiores sem oostegitos foram considerados machos. Indivíduos de classes de tamanho inferiores foram considerados jovens. Fêmeas carregando ovos e embriões foram consideradas ovígeras (JACоBucCl et al. 2002).

Considerou-se a fecundidade como o número de ovos contidos no marsúpio de fêmeas ovígeras, sendo a avaliação de fecundidade realizada somente com as fêmeas que estavam com os marsúpios intactos ou fechados. Para essas fêmeas procedeuse à contagem total dos ovos e à medição do comprimento cefálico. A utilização do comprimento cefálico dos anfípodes se justifica pela facilidade de medição e pelo fato deste geralmente apresentar elevada correlação com o comprimento total do corpo em gamarídeos (LOPES \& M ASUNARI 2004). O volume dos ovos foi cal culado utilizando-se a expressão: $V=\pi / 6\left(d_{1} / 2+d_{2} / 2\right)^{3}$, sendo $d_{1}$ o maior diâmetro e $d_{2}$ o menor (Leite 1996a). 
Calculou-se a proporção mensal de machos para fêmeas (razão sexual) para cada espécie, dos indivíduos acima da classe de tamanho $1,4 \mathrm{~mm}$.

Os exemplares coletados durante o estudo encontram-se depositados na coleção de referência do Laboratório de Interações em Comunidades Marinhas (LICOMAR), Departamento de Zoologia, Instituto de Biologia, Universidade Estadual de Campinas.

Análise dos dados

Para avaliar diferenças mensais no peso seco de $\mathrm{S}$. filipendula e de suas epífitas, foi utilizada ANOVA unifatorial, seguida do teste a posteriori de Newman-Keuls.

A relação entre o comprimento cefálico e o número de ovos contidos no marsúpio das fêmeas ovígeras foi avaliada através do coeficiente de correlação de Spearman. Foi calculada a média e o desvio padrão do número e do volume dos ovos das fêmeas ovígeras, que foram comparados entre espécies utilizando-se Análise de Variância. O período reprodutivo e o período de recrutamento das espécies foram avaliados, respectivamente, através da freqüência relativa de fêmeas ovígeras e de jovens ao longo do ano. A razão sexual foi testada em relação à proporção 1:1 utilizando-se o teste de Qui-quadrado $\left(\chi^{2}\right)$. Para todas as análises estatísticas utilizou-se o programa BioEstat 3.0 (Ayres et al. 2003), considerando-se o nível de significância $\alpha=0,05$.

\section{RESULTADOS}

Durante o período de coletas a menor temperatura média superficial da água foi registrada no mês de julho $(20,8$ o $C)$ e a maior em dezembro $(29,3 \circ \mathrm{C})$.

Houve variação no peso das frondes coletadas, notandose uma redução significativa somente em dezembro de 2000 e janeiro de 2001. As epífitas também apresentaram variação de peso seco com o mês de dezembro apresentando o valor médio significativamente mais el evado (Fig. 1).

Foram identificados 3505 indivíduos, sendo 1738 pertencentes à espécie A. ramondi, 929 à C. filosa e 838 à S. pelagica. As densidades máximas de $A$. ramondi e $C$. filosa foram registradas em janeiro de 2001 e de S. pelagica em novembro de 2000 (Fig. 2).

Os histogramas das classes de tamanho das três espécies de Ampithoidae estão evidenciados nas figuras 3 a 5. Durante todo o período, notou-se a predominância de jovens ( $<1,4 \mathrm{~mm}$ ) para as três espécies, vindo a seguir as fêmeas e os machos. Nos meses de dezembro de 2000, abril e maio de 2001 ocorreram somente jovens de A. ramondi (Fig. 3). Para C. filosa foram observados machos, fêmeas e jovens em todos os períodos de coleta (Fig. 4). Nos meses de dezembro de 2000, fevereiro, março, abril e maio de 2001 não foi registrada a presença de S. pelagica (Fig. 5). Os maiores indivíduos das espécies Ampithoe ramondi e
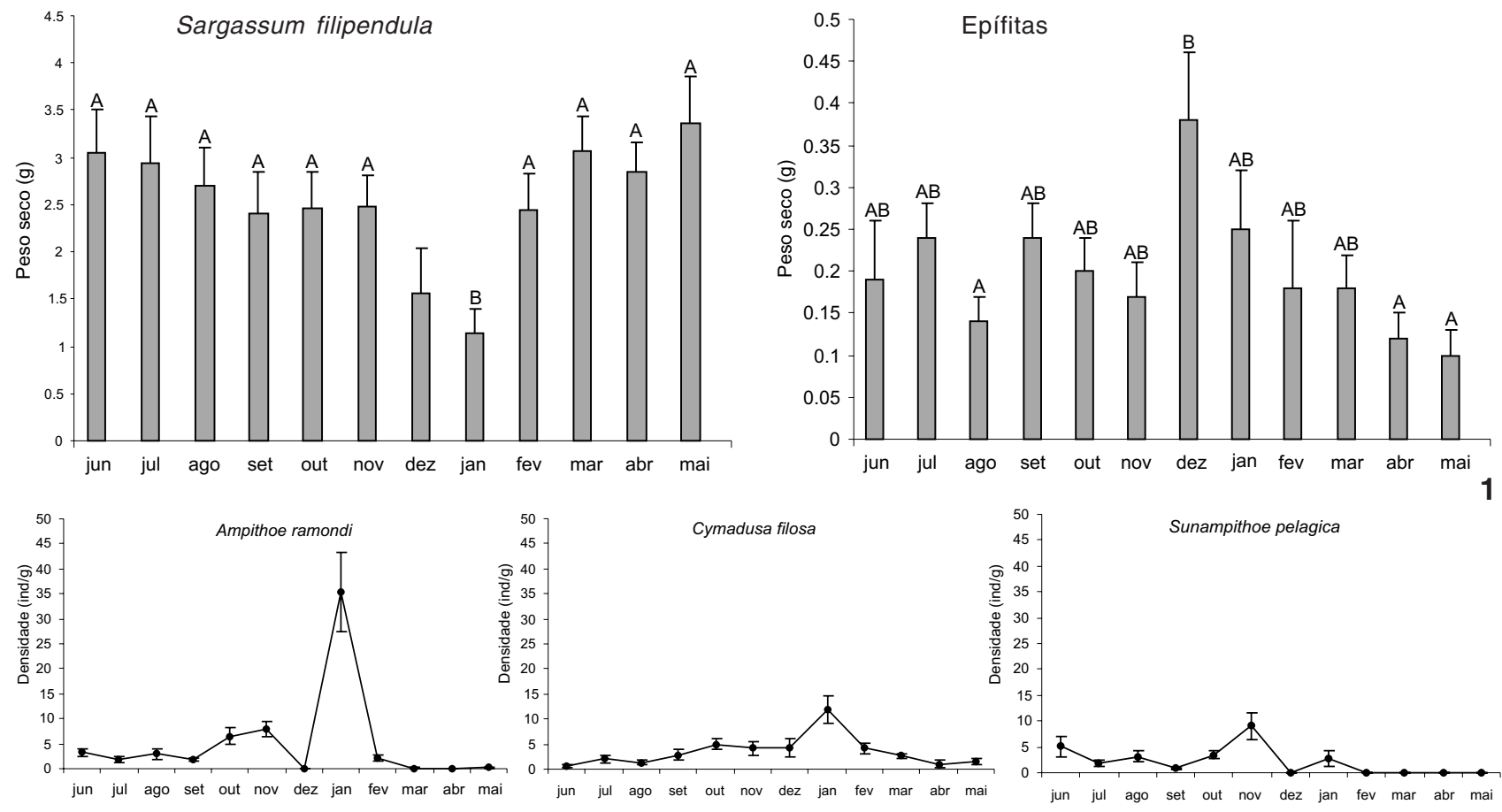

Figuras 1-2. (1) Peso seco em gramas ( \pm erro padrão) das frondes de Sargassum filipendula e das epífitas. Barras com a mesma letra indicam ausência de diferença significativa. (2) Densidade média ( \pm erro padrão) (número de indivíduos/grama de peso seco de Sargassum filipendula) dos anfípodes ampitoídeos, associados à Sargassum filipendula na praia da Fortaleza, São Paulo. 

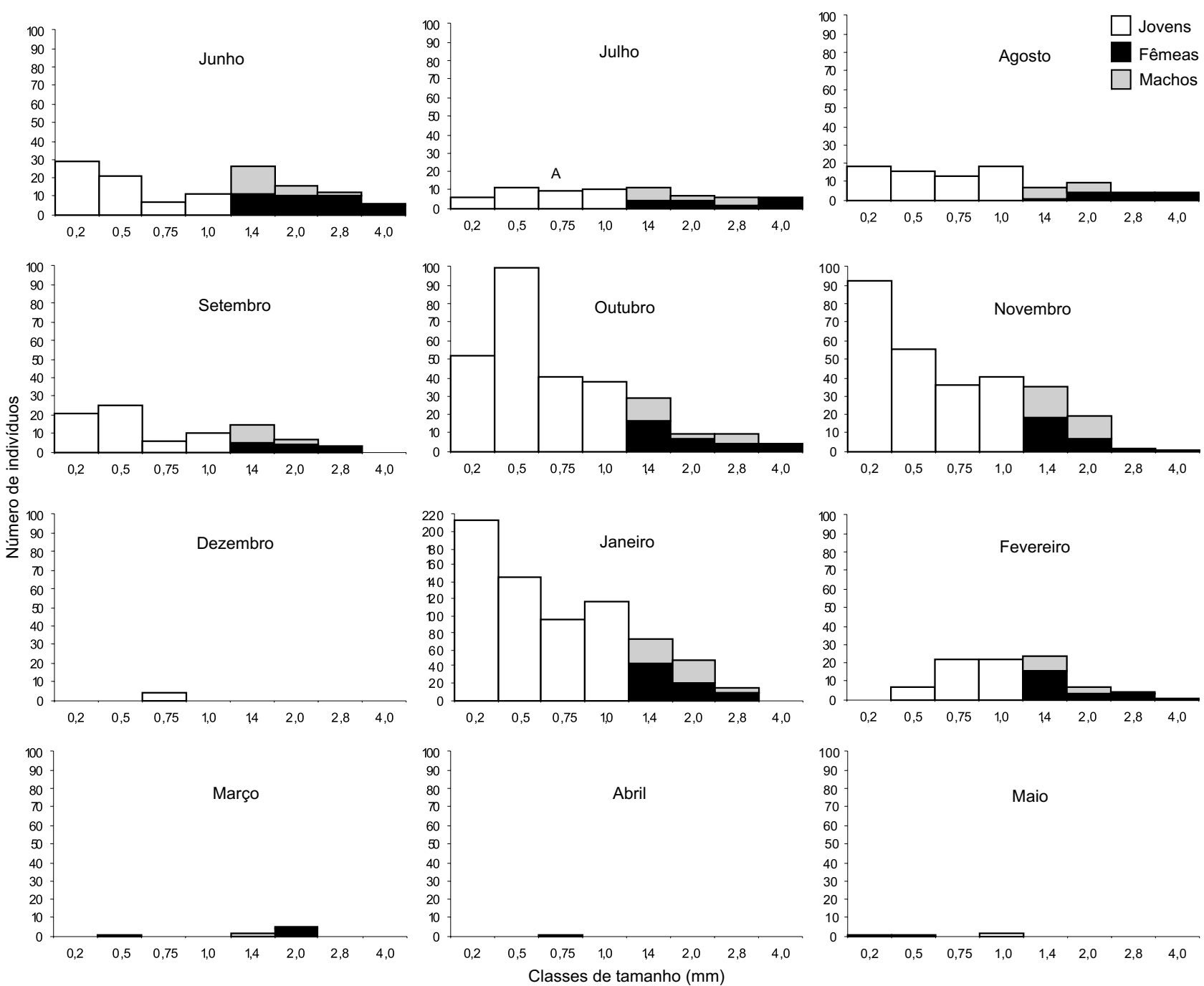

Figura 3. Histogramas mensais de freqüência de tamanho de fêmeas, machos e jovens de Ampithoe ramondi, associados a Sargassum filipendula na praia da Fortaleza, São Paulo.

C. filosa ocorreram na classe de tamanho de $4,0 \mathrm{~mm}$, enquanto S. pelagica foi registrada até a classe de 2,8 $\mathrm{mm}$ (Fig. 5).

Fêmeas ovígeras de A. ramondi e C. filosa estiveram presentes em quase todos os meses de amostragem (Fig. 6), enquanto indivíduos jovens das três espécies foram registrados em todos os períodos em que os anfípodes foram coletados (Fig. 7).

Foi analisado um total de 62 fêmeas ovígeras com suas bolsas incubadoras intactas durante todo o período, sendo 33 de A. ramondi, 16 deC. filosa e 13 deS. pelagica. Asfêmeas ovígeras das três espécies apresentaram diferenças quanto ao número mínimo e máximo de ovos no marsúpio. Ampithoe ramondi apresentou entre 9 e 59; C. filosa: entre 15 e 69 e S. pelagica entre 10 e 22. Foi observada diferença no número médio de ovos entre as espécies (A. ramondi: $n=20,24 \pm 12,38$; C. filosa: $n=29,63 \pm$ 17,04; S. pelagica: $n=14,92 \pm 3,84$ ) sendo a fecundidade de $C$. filosa significativamente maior do que a das demais espécies (ANOVA: $\mathrm{F}=19,37$; g.l. $=2 ; \mathrm{p}<0,05$ ). $\mathrm{O}$ volume dos ovos das espécies também foi significativamente diferente (ANOVA: $F=$ 328,31; g.l. $=2 ; p<0,05)$. Cymadusa filosa apresentou ovos com maior volume $\left(V=0,034 \pm 0,008 \mathrm{~mm}^{3}\right)$, seguida de $A$. ramondi $\left(V=0,033 \pm 0,010 \mathrm{~mm}^{3}\right)$ e S. pelagica $\left(V=0,016 \pm 0,005 \mathrm{~mm}^{3}\right)$.

Verificou-se pequena dependência do número de ovos contidos no marsúpio de $A$. ramondi em relação ao comprimento cefálico da fêmea $\left(r^{2}=0,27 ; p<0,05 ; n=33\right)$. Cymadusa filosa ( $\left.r^{2}=0,17 ; n s ; n=16\right)$ e S. pelagica $\left(r^{2}=0,10 ; n s ; n=13\right)$ não apresentaram relações significativas.

A razão sexual total, considerando-se anfípodes de todas as amostras, diferiu de $1: 1$ para $A$. ramondi $\left(1: 1,2 ; \chi^{2}=4,37 ;\right.$ g.l. $=1 ; p<0,05)$, não havendo diferença na proporção de machos:fêmeas para C. filosa $\left(1: 0,8 ; \chi^{2}=2,80 ;\right.$ g.l. $=1$; ns) e . 

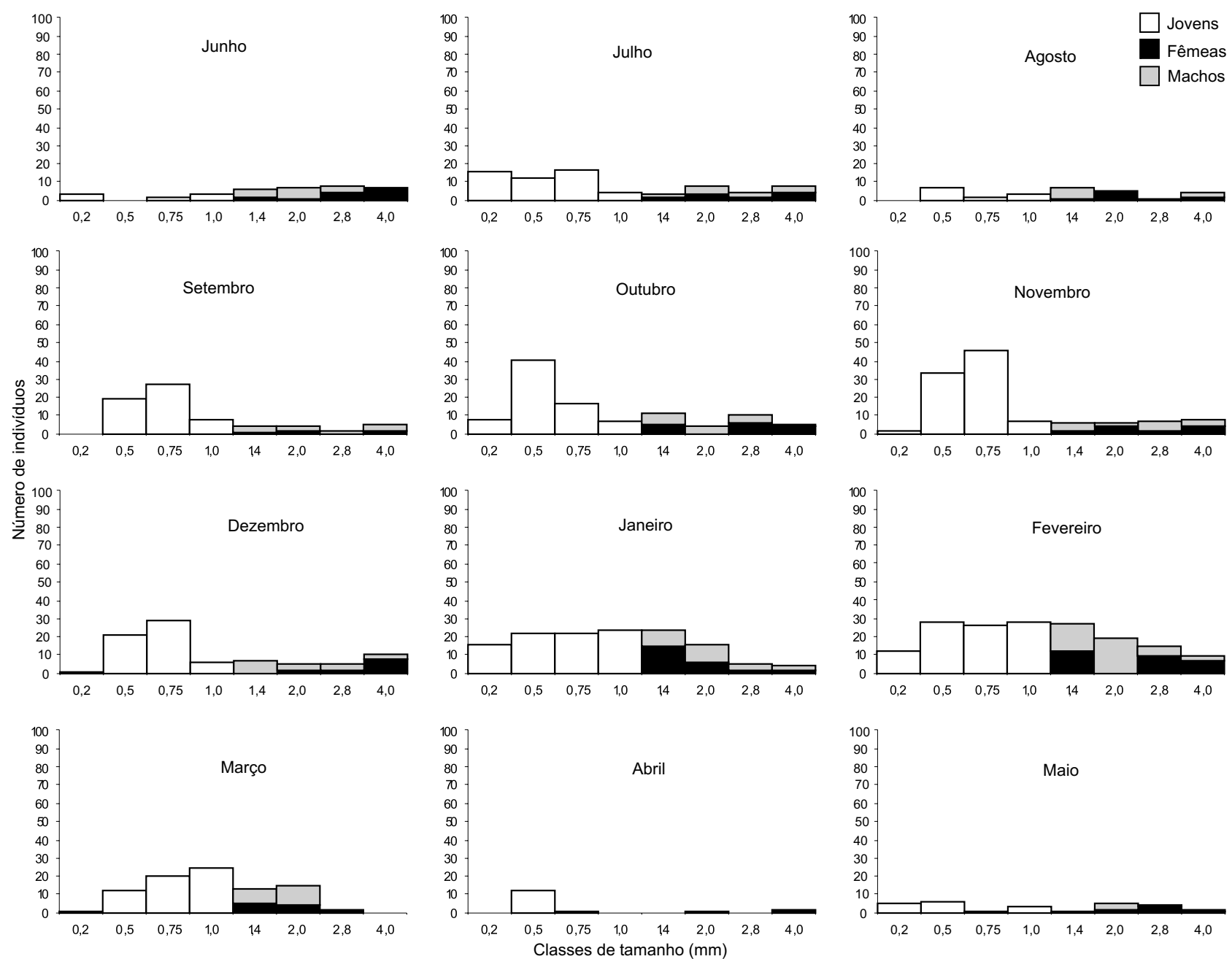

Figura 4. Histogramas mensais de freqüência de tamanho de fêmeas, machos e jovens de Cymadusa filosa, associados à Sargassum filipendula na praia da Fortaleza, São Paulo.

pelagica $\left(1: 1 ; \chi^{2}=0,005 ;\right.$ g.l. $\left.=1 ; n s\right)$. Ao longo do período de amostragem, A. ramondi apresentou a proporção machos:fêmeas variando de 1:0,7 a 1:1,6, C. filosa de 1:0,5 a 1:2,0 e S. pelagica de $1: 0,7$ a $1: 1,2$ (Fig. 8).

\section{DISCUSSÃO}

Os dados obtidos indicam grande variação ao longo do ano na densidade das três espécies de ampitoídeos investigadas. Sabe-se que a variação temporal da fauna de gamarídeos do fital pode estar relacionada a modificações de fatores ambientais locais como temperatura, hidrodinamismo, sedimentação, bem como mudanças sazonais na pressão de predação, suprimento de alimento e atividade reprodutiva (EDGAR 1990, DUBIASKI-SILVA \& MASUNARI 1995).

Os picos de densidade de A. ramondi e C. filosa ocorreram em janeiro, mês no qual o peso seco médio de S. filipendula atingiu seu valor mínimo. A redução no tamanho das frondes de S. filipendula no verão coincide com o aumento da quantidade de al gas epífitas. Essas epífitas podem gerar efeitos del etérios sobre as macrófitas que Ihes servem de suporte, pois modificam o microambiente no entorno das macrófitas, aumentando o sombreamento e a possibilidade de fragmentação e interceptando nutrientes da coluna d'água (ORTH \& VAN Montfrans 1984, Buschmann \& Gomez 1993, Williams \& Ruckelshaus 1993).

O elevado número de anfípodes por fronde em janeiro pode ser resultado simplesmente da redução do substrato disponível. Este parece ser o caso de C. filosa, que não apresentou grande variação no número total de indivíduos entre os meses de novembro e janeiro. Por outro lado, A. ramondi apresentou grande aumento no número total de indivíduos, particularmente de jovens, indicando que houve elevado investimento reprodutivo no período anterior. Este fato pode estar relacio- 

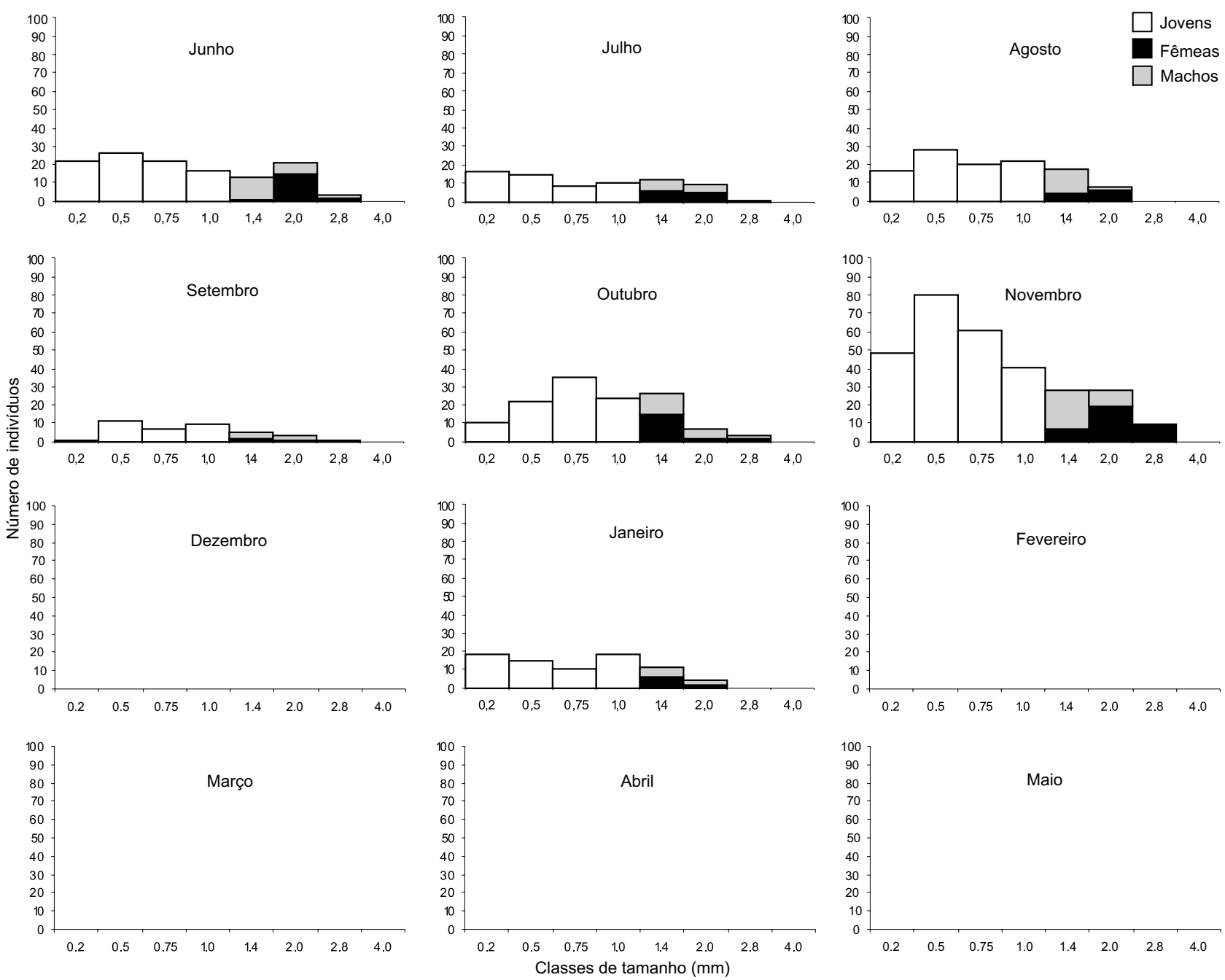

Figura 5. Histogramas mensais de freqüência de tamanho de fêmeas, machos e jovens de Sunampithoe pelagica, associados a Sargassum filipendula na praia da Fortaleza, São Paulo.

nado ao grande aumento na disponibilidade de epífitas em dezembro. Rápidos aumentos populacionais de anfípodes do fital relacionados ao crescimento de algas epífitas em áreas abrigadas já foram relatados (EDGAR \& MOORE 1986). Essas al gas poderiam estar servindo de alimento e/ou material para a construção de tubos para os ampitoídeos.

A redução brusca na densidade de A. ramondi e o desaparecimento de $\mathrm{S}$. pelagica em dezembro podem estar relacionados às condições locais de el evada temperatura e reduzida movimentação da água no período (observação pessoal). Nestas circunstâncias, há possibilidade de redução da concentração de oxigênio dissolvido na água, ocasionando mortalidade em espécies menos tolerantes (ConLAN 1994).

A partir de fevereiro nota-se uma redução acentuada não apenas da densidade de epífitas, o que seria esperado em fun- ção do aumento do peso médio das frondes de S. filipendula, mas também do número absoluto de indivíduos, particularmente de $\mathrm{A}$. ramondi e S. pelagica. Esta última espécie não foi amostrada e este fato pode ser explicado pela redução progressiva do peso das algas epífitas, representando restrição de abrigo e alimento para os anfípodes (LeITE \& TURRA 2003).

Sunampithoe pelagica apresenta menores dimensões e, aparentemente menor potencial reprodutivo (considerando-se que possui menor fecundidade média) em relação aos outros ampitoídeos, como também observado por LEITE (1996a). Deste modo, se considerarmos a disponibilidade de epífitas como fator limitante às populações de ampitoídeos do local , S. pelagica estaria em situação competitivamente inferior. Como subsídio a esta hipótese, há relatos de comportamentos agonísticos entre ampitoídeos em condições de superpovoamento (Brawley 


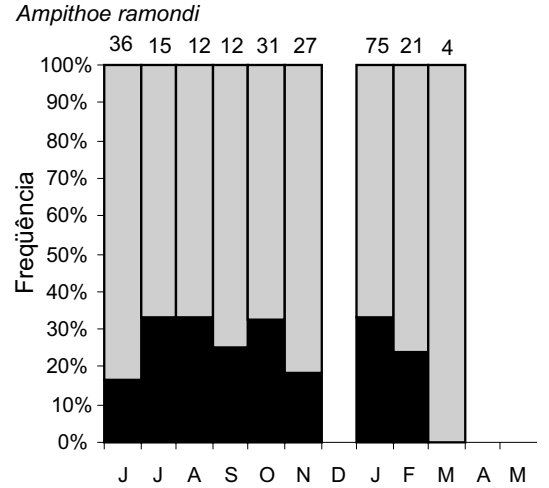

Ampithoe ramondi
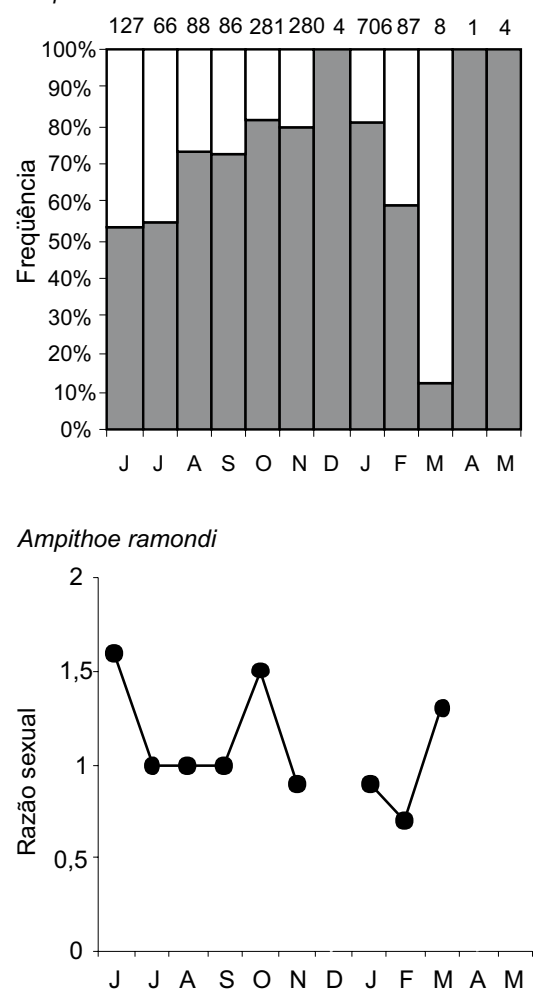

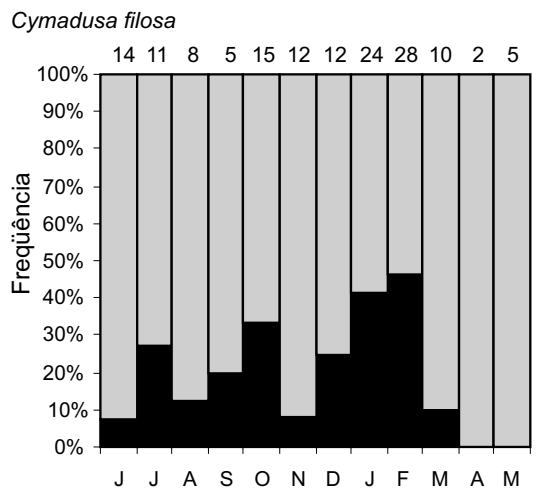

Cymadusa filosa
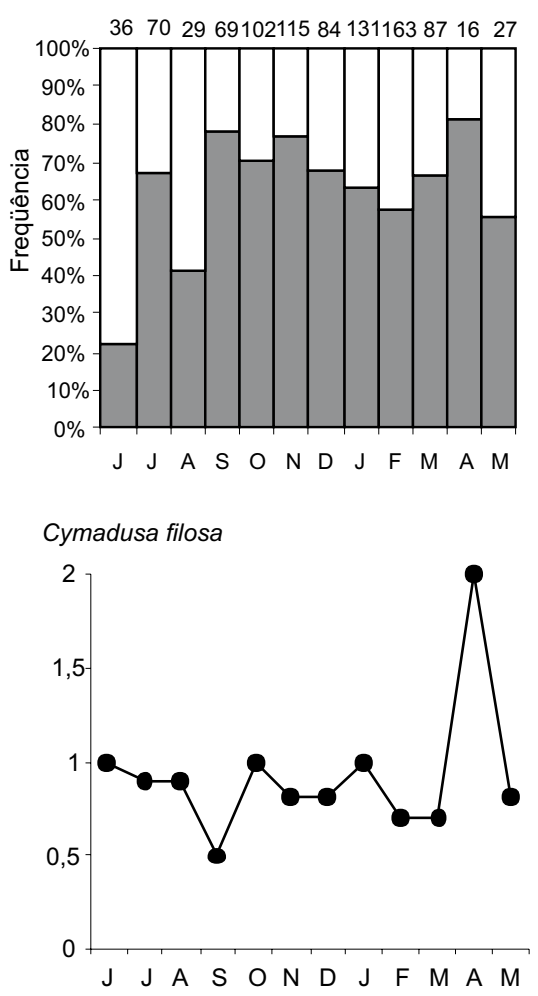
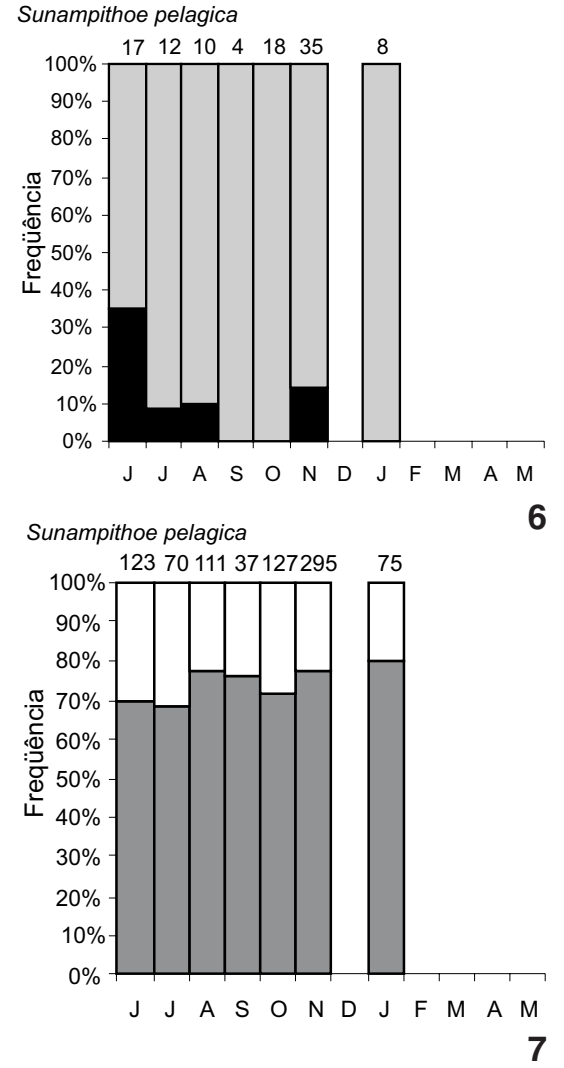

Sunampithoe pelagica

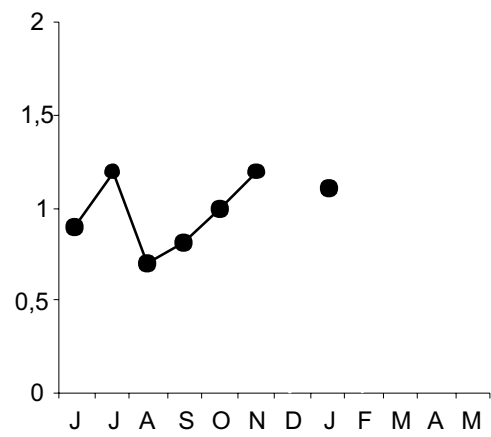

Figuras 6-8. (6) Freqüência de fêmeas ovígeras (em preto) e não ovígeras (em cinza) dos anfípodes ampitoídeos, associados a Sargassum filipendula na praia da Fortaleza, São Paulo; (7) freqüência de jovens (em cinza) e adultos (em branco) dos anfípodes ampitoídeos, associados à Sargassum filipendula na praia da Fortaleza, São Paulo; (8) razão sexual (macho:fêmea) de Ampithoe ramondi, Cymadusa filosa e Sunampithoe pelagica, associados à Sargassum filipendula na praia da Fortaleza, São Paulo.

\& Adey 1981). Além disso, BarReto (1999) registrou diferenças significativas de ocorrência de ampitoídeos em algas da Praia da Fortaleza, com S. pelagica ocupando preferencialmente Galaxaura marginata (J. Ellis \& Solander) J. V. Lamouroux, 1816 em relação a S. filipendula. Com a limitação de recursos, S. pelagica poderia estar migrando para G. marginata.

Outro fator regulador importante das populações de ampitoídeos pode ser a predação por peixes. No local ocorrem ao longo detodo o ano peixes bentívoros que utilizam anfípodes e outros organismos do fital como alimento, fato também observado por Tararam \& WaKabara (1982) em Itanhaém. Outras espécies de peixes visitantes com comportamento de forrageio solitário e em cardume foram observadas no local, durante o inverno (observação pessoal). Densidades elevadas de preda-

Revista Brasileira de Zoologia 23 (4): 1207-1216, dezembro 2006 
dores de anfípodes já foram registradas no inverno em outras áreas do litoral sudeste brasileiro (WAKABARA et al. 1996), sendo consideradas importante fator regulador dos anfípodes associados a Bryocladia thyrsigera (J. Agardh) F. Schmitz, 1901 no litoral sul de São Paulo (VAlérIo-BerARdo \& Flynn 2002).

Como indicativo da contribuição da atividade reprodutiva das espécies para a variação da abundância, observa-se que os maiores picos de densidade tanto de $\mathrm{A}$. ramondi como $\mathrm{S}$. pelagica se devem ao maior número de jovens $(<1,4 \mathrm{~mm})$ presentes nas amostras. A presença de jovens em todos os meses e de fêmeas ovígeras em quase todos os períodos de amostragem indica também que $A$. ramondi e $C$. filosa apresentam reprodução e recrutamento contínuos. Ambas as espécies seguem um padrão típico de anfípodes de regiões tropicais e subtropicais que se caracterizam por apresentarem multivoltinismo e recrutamento ao longo de todo o ano (SAINTE-MARIE 1991, ValérioBERARDO \& FlYNN 2004). No caso de S. pelagica foram registradas fêmeas com ovos no inverno e em novembro, mês com maior número de indivíduos da espécie.

O pequeno número de indivíduos de maior porte (sempre abaixo de $7 \%$ do total de organismos coletados em cada período) pode estar relacionado à predação seletiva de peixes sobre os gamarídeos. É bem conhecida a ação dos peixes sobre a fauna do fital tanto direta como indiretamente, isto é, peixes herbívoros que ao se alimentarem da alga acabam comendo também os anfípodes a ela associados (Robertson \& Howard 1978, Nelson 1979). Segundo EdGAR (1983b), a mortalidade de anfipodes ampitoídeos de pequenas dimensões seria causada principalmente por fatores ambientais e/ou predação por pequenos predadores não seletivos do fital, enquanto predadores visuais sel etivos, representados basicamente por peixes, seriam capazes de reduzir consideravelmente a ocorrência de anfípodes de maiores dimensões.

O tamanho do ovo está relacionado com a energia estocada para posterior desenvolvimento (GIANGRANDE et al. 1994). Entre os crustáceos incubadores o tamanho do ovo é inversamente correlacionado com o número de ovos em qualquer tamanho de fêmea (Steele \& Steele 1975). A diferença significativa no número médio de ovos de $C$. filosa em relação às outras espécies $\mathrm{e}$ do volume dos ovos são indicativos de que adotam diferentes estratégias de alocação energética para reprodução.

Outro fator relacionado com diferentes estratégias, diz respeito à duração do período embrionário (CARDOSO \& VELOSO 2001). Ovos de tamanhos diferentes se desenvolvem em tempos diferentes, isto é, os menores ovos eclodem antes que os maiores (Steele \& Steele 1975), portanto os ovos de S. pelagica tendo em vista seu menor volume, se desenvolveriam mais rapidamente que os das outras duas espécies simpátricas. LeITE (1996a) observou que o volume de ovo de A. ramondi era o menor entre os ampitoídeos estudados. A diferença observada entre os dois estudos pode ser atribuída a diferenças no tamanho das fêmeas coletadas, já que existe uma relação entre o tamanho do corpo da fêmea e o número e tamanho dos ovos (VAN DoLAH \& BIRD 1978, SAInTE-M ARIE 1991). Além disso, populações de áreas dis- tintas submetidas a diferentes condições de temperatura, hidrodinamismo, salinidade, disponibilidade de alimento, pressão de predação e competição, podem apresentar variação em seus parâmetros reprodutivos, incluindo número, volume e taxa de eclosão de ovos (Morino 1978, SteEle 1981).

Para os malacóstracos, em geral, o tamanho da ninhada e o diâmetro do embrião estão positivamente correlacionadoscom o tamanho do corpo da fêmea (SAINTE-M ARIE 1991), fato já observado para várias espécies de anfípodes gamarídeos associados a Sargassum (LEITE 1996a, c). Esta relação foi observada somente em A. ramondi. A ausência de relação significativa entre o comprimento da cabeça e o número de ovos para as outras duas espécies, resultado distinto daquele registrado por LeITE (1996a), pode ser devida ao pequeno número de fêmeas ovígeras avaliadas com o marsúpio intacto.

Segundo a Teoria de Fisher da razão sexual, a sel eção natural favoreceo investimento parental em prolescom número igual de machos e fêmeas. O desvio da proporção 1:1 pode estar relacionado a fatores dependentes do ciclo de vida das espécies $\left(\mathrm{H}_{\mathrm{A}-}\right.$ MILTON 1967), como diferenças na taxa de mortalidade, Iongevidade, razão de crescimento, comportamento e utilização de habitat entre os sexos (Wenner 1972, Gable \& Crooker 1977). Diferenças de mortalidade podem ocorrer em função de maior susceptibilidade de um dos sexos à predação, devido ao comportamento reprodutivo das espécies. LeITE et al. (2003) explicaram a menor proporção de machos em populações do tanai dáceo Kalliapseudes schubarti em função de seu deslocamento à procura de fêmeas torná-los mais vulneráveis à predação. Embora sejam necessários estudos comportamentais, situação semel hante poderia estar ocorrendo com A. ramondi.

Ao estudar a macrofauna associada a Sargassum stenophylum, TANAKA \& Leite (2003) ressaltam que as características da história natural de diferentes grupos funcionais e espécies, particularmente dos anfípodes gamarídeos, podem ser importantes para explicar os padrões de distribuição dessas espécies. Os resultados obtidos no presente estudo reforçam a importância de investigações acerca da biologia dos anfípodes em nível específico, já que espécies da mesma família, de um mesmo grupo funcional (tubícolas) e de hábito alimentar semelhante (herbívoro, herbívoro-detritívoro) podem apresentar diferenças importantes em parâmetros populacionais como densidade, razão sexual, fecundidade e volume de ovos.

\section{AGRADECIMENTOS}

À Fundação de Amparo à Pesquisa do Estado de São PauIo (FAPESP) pela bolsa de doutorado concedida ao primeiro autor (Proc. no 99/11325-9). Ao Dr. Alexander Turra e ao Ms. Arthur Z. Güth pelo auxílio nas coletas.

\section{REFERÊNCIAS BIBLIOGRÁFICAS}

Ayres, M; M. Ayres JR; D.L. Ayres \& A.S. Santos. 2003. BioEstat 3.0: aplicações estatísticas nas áreas das Ciências Bioló-

Revista Brasileira de Zoologia 23 (4): 1207-1216, dezembro 2006 
gicas e Médicas. Belém, Sociedade Civil Mamirauá, MCTCNPq, Conservation International, XII+291p.

BARNARD, J.L. \& G.S. KARAMAN. 1991a. The families and genera of marine gammaridean Amphipoda (except marine gammaroids). Records of the Australian Museum Suplement, Sydney, 13 (1): 1-417.

BARNARD, J.L. \& G.S. KaRAMAN. 1991b. The families and genera of marine gammaridean amphipoda (except marine gammaroids). Records of the Australian Museum Suplement, Sydney, 13 (2): 419-866.

BARREto, C.C. 1999. Heterogeneidade espacial do habitat e diversidade específica: implicações ecológicas e métodos de mensuração. Oecologia Brasiliensis, Rio de Janeiro, 84: 158-163.

BousfieLD, E.E. 1973. Shallow-water gammaridean Amphipoda of New England. London, Comstock Publishing, Cornell University Press, 312p.

BraWLEY, S.H. \& W.H. AdeY. 1981. The effects of micrograzers on algal community structure in a coral reef microcosm. Marine Biology, Berlim, 61: 167-178.

Buschmann, A.H. \& P. Gomez. 1993. Interaction mechanisms between Gracilaria chilensis (Rodophyta, Gigartinales) and epiphytes. Hydrobiologia, Dordrecht, 261: 345-351.

CARDoso, R.S. \& V.G. Veloso 2001. Embryonic development and reproductive strategy of Pseudorchestoidea brasiliensis (Amphipoda, Talitridae) at Prainha Beach, Brazil. Journal of Natural History, London, 35: 201-211.

Conlan, K.E.1994. Amphipod crustaceans and environmental disturbance: a review. Journal of Natural History, London, 28: 519-554.

DUBIASKI-SILVA, J. \& S. MaSUnARI. 1995. Ecologia populacional dos Amphipoda (Crustacea) dos fitais de Caiobá, Matinhos, Paraná, Brasil. Revista Brasileira de Zoologia, Curitiba, 12 (2): 373-396.

DufFY, J.E. 1990. Amphipods on seaweeds: partners or pests? Oecologia, Berlin, 83: 267-276.

EdGAR, G.J. 1983a. The ecology of south-east Tasmanian phytal animal communities. I. Spatial organization on a local scale. Journal of Experimental Marine Biology and Ecology, Amsterdam, 70: 129-157.

EdGAR, G.J. 1983b. The ecology of south-east Tasmanian phytal animal communities. IV. Factors affecting the distribution of ampithoid amphipods among algae. Journal of Experimental Marine Biology and Ecology, Amsterdam, 70: 205-225.

EDGAR, G.J. 1990. Population regulation, population dynamics and competition amongst mobile epifauna associated with seagrass. Journal of Experimental Marine Biology and Ecology, Amsterdam, 144: 205-234.

EdGAR, G.J. \& P.G. MoORE. 1986. Macro-al gae as habitats for motile macrofauna. Monografias Biologicas, Barcelona, 4: 255-277.

GABle, M.F. \& R.A. Crooker. 1977. The salt marsh amphipod Gammarus palustris Bousfield, 1969 at the northern limit of its distribution. I. Ecology and life cycle. Estuarine, Coastal and Shelf Science, Amsterdam, 5 (1): 123-134.
Giangrande, A.; S. Geraci \& G. Belmonte. 1994. Life cycles and life history diversity in marine invertebrates and the implications in community dynamics. Oceanography and Marine Biology, Aberdeen, 32: 305-333.

HAMILTON, W.O. 1967. Extraordinary sex ratio. Science, Washington, 156: 44-488.

Holmlund M.; C.H. Peterson \& M.E. Hay. 1990. Does algal morphology affect amphipod susceptibility to fish predation? Journal of Experimental Marine Biology and Ecology, Amsterdam, 139: 65-83.

Jacobucci, G.B.; D. Moretti; E.M. Silva \& F.P.P. Leite. 2002. Caprellid amphipods on Sargassum cymosum (Phaeophyta): Depth distribution and population biology. Nauplius, Botucatu, 10 (1): 27-36.

Jacoвuccl, G.B. \& F.P.P. Leite. 2002. Distribuição vertical e flutuação sazonal da macrofauna vágil associada a Sargassum cymosum C. Agardh, na praia do Lázaro, Ubatuba, São PauIo, Brasil. Revista Brasileira de Zoologia, Curitiba, 19 (Supl. 1): 87-100.

LeITE, F.P.P. 1996a. Fecundidade de sete espécies de gamarídeos (Crustacea, Amphipoda, Gammaridea) associados à alga Sargassum cymosum. Iheringia, Série Zoologia, Porto Alegre, 80: 39-45.

LeITE, F.P.P. 1996b. Alterações morfológicas dos estádios de crescimento de Amphilocus neapolitanus, Della Valle (Gammaridea, Amphilochidae) e Amphithoe ramondi (Gammaridea, Ampithoidae), anfípodes associados à alga Sargassum cymosum. Revista Brasileira de Zoologia, Curitiba, 13 (1): 47-60.

LeITE, F.P.P. 1996c. Crescimento e reprodução de Hyale media Dana (Amphipoda, Gammaridae, Hyalidae) associada a Sargassum cymosum C. Agardh. Revista Brasileira de Zoologia, Curitiba, 13 (3): 597-606.

Leite, F.P.P. \& A. TurRa. 2003. Temporal variation in Sargassum biomass, Hypnea epiphytism and associated fauna. Brazilian Archives of Biology and Technology, Curitiba, 46 (4): 665671.

LeITE, F.P.P.; Y. Wakabara \& A.S. Tararam. 1986. On the morphological variation of the oostegites of gammaridean species. Crustaceana, Leiden, 51 (1): 72-94.

Leite, F.P.P.; A. Turra \& E.C.F SouzA. 2003. Population biology and distribution of the tanaid Kalliapseudes schubarti MañéGarzon, 1949, in an intertidal flat in southeastern Brazil. Brazilian Journal of Biology, São Carlos, 63 (3): 469-479.

LEITE, F.P.P. \& A.Z. GüTH. 2003. Variações morfológicas dos estágios pós-marsupiais de Sunampithoe pelagica Milne-Edwards (Crustacea, Amphipoda, Gammaridea, Ampithoidae) da fauna de Sargassum cymosum C. Agardh. Revista Brasileira de Zoologia, Curitiba, 20 (1): 65-73.

LeITE, F.P.P.; A.Z. GÜTH \& G.B. JACOBUCCI. 2000. Temporal comparison of gammaridean amphipods of Sargassum cymosum on two rocky shores in southeastern Brazil. Nauplius, Botucatu, 8 (2): 227-236.

Lopes, O.L. \& S. MASUnARI. 2004. Biologia reprodutiva de Tali- 
troides topitotum (Burt) (Crustacea, Amphipoda, Talitridae) na Serra do Mar, Guaratuba, Paraná, Brasil. Revista Brasileira de Zoologia, Curitiba, 21 (4): 755-759.

Morino, H. 1978. Studies on the Talitridae (Amphipoda, Crustacea) in Japan III. Life history and breed activity of Orchestia platensis Kröyer. Publications of Seto Marine Biology Laboratory, Wakayama, 24: 245-267.

MYERS, A.A. 1985. Shallow-water, coral reef and mangrove Amphipoda (Gammaridea) of Fiji. Records of the Australian Museum Suplement, Sydney, 5: 1-143.

NeLSON, W.G. 1979. Experimental studies of selective predation on amphipods: consequences for amphipod distribution and abundance. Journal of Experimental Marine Biology and Ecology, Amsterdam, 38: 225-245.

Norton, T.A. \& M.R. Benson. 1983. Ecological interactions between the brown seaweed Sargassum muticum and its associated fauna. Marine Biology, Leiden, 75: 169-177.

ORTH, R.J. \& J. VAN MONTFRANS.1984. Epiphyte-seagrass relationship with an emphasis on the role of micrograzing: A review. Aquatic Botany, Amsterdam, 18: 43-69.

Paula, E.J. \& E.C. Oliveira Filho. 1980. Phenology of two populations of Sargassum cymosum (Phaeophyta - Fucales) of São Paulo State coast, Brazil. Boletim de Botânica, São Paulo, 8: 21-39.

RoberTSON, A.I. \& R.K. HowARD. 1978. Diel trophic interactions between vertically migrating zooplankton and their fish predators in an eelgrass community. Marine Biology, Leiden, 48: 207-213.

Ruffo, S. 1982. The Amphipoda of the Mediterranean, Part 1, Gammaridea (Acanthonotozomatidaeto Gammaridae). Memoires d'Institute Oceanographique, Monaco, 13: 1-364.

SAINTE-M ARIE, B. 1991. A review of the reproductive bionomics of aquatic gammaridean amphipods: variation of life history traits with latitude, depth, salinity and superfamily. Hydrobiologia, Dordrecht, 223: 189-227.

SALA, E. 1997. The role of fishes in the organization of a Mediterranean sublitoral community. I: Epifaunal communities. Journal of Experimental Marine Biology and Ecology, Amsterdam, 212: 45-60.

Steele, V.J. 1981. The effect of photoperiod on the reproductive cycle of Gammarus lawrencianus Bousfield. Journal of Experimental Marine Biology and Ecology, Amsterdam, 53: 1-7.

Steele, D.H. \& V.J. Steele. 1975. The biology of Gammarus (Crustacea, Amphipoda) in the northwestern Atlantic. XI. Comparison and discussion. Canadian Journal of Zoology, Ottawa, 53: 1116-1126.

SzÉCHY, M.T.M. \& E.J. PAuLA. 2000. Padrões estruturais quantitativos em bancos de Sargassum (Phaeophyta, Fucales) do li- toral dos estados do Rio de Janeiro e São Paulo, Brasil. Revista Brasileira de Botânica, São Paulo, 23 (2): 121-132.

TANAKA, M.O. \& F.P.P. Leite. 1998. The effect of sieve mesh size on the abundance and composition of macrophyte-associated macrofaunal assemblages. Hydrobiologia, Dordrecht, 389: 21-28.

TANAKA, M.O. \& F.P.P. LeITE. 2003. Spatial scaling in the distribution of macrofauna associated with Sargassum stenophylum (Mertens) martius: analyses of faunal groups, gammarid life habits, and assemblage structure. Journal of Experimental Marine Biology and Ecology, Amsterdam, 293 (1): 1-22.

TANAKA, M.O. \& F.P.P. LEITE. 2004. Distance effects on short-term recolonization of Sargassum stenophyllum by mobile epifauna, with an analysis of gammarid life habits. Journal of the Marine Biological Association of the United Kingdom, Plymouth, 84 (5): 901-910.

TARARAM, A.S. \& Y. WAKABARA. 1981. The mobile fauna- especially Gammaridea - of Sargassum cymosum. Marine Ecology Progress Series, Amelinghausen, 5: 157-163.

TARARAM, A.S. \& Y. Wakabara. 1982. Notes on the feeding of Blennius cristatus Linnaeus from a rocky pool of Itanhaém, São Paulo state. Boletim do Instituto Oceanográfico, São Paulo, 31 (2): 1-3.

Valério-Berardo, M.T. \& M.N. Flynn. 2002. Composition and seasonality of an amphipod community associated to the al gae Bryocladia thyrsigera. Brazilian Journal of Biology, São Carlos, 62 (4A): 735-742.

Valério-Berardo, M.T. \& M.N. Flynn. 2004. Population biology of Hyale nigra (Haswell, 1979) (Amphipoda, Hyalidae) associated to Bryocladia thyrsigera (J. Agardh) at Peruíbe, Itanhaém beach, southeastern Brazil. Nauplius, Botucatu, 12 (1): 1-10.

VAN DOLAH, R.F. \& E. BIRD. 1978. Factors regulating the distribution and population dynamics of the amphipod Gammarus palustris in an intertidal salt marsh community. Ecological Monographs, Ithaca, 48: 191-217.

Wakabara, Y.; A.S. TARARAm \& A.M. TAKedA. 1983. Comparative study of the amphipod fauna living on Sargassum of two Itanhaém shores, Brazil. Journal of Crustacean Biology, Kansas, 3 (4): 602-607.

Wakabara, Y.; M. Flynn \& A.S. Tararam. 1996. Ingestion and selection of suprabenthic crustaceans by small-sized fishes in a lower saltmarsh system. Revista Brasileira de Oceanografia, São Paulo, 44: 89-103.

Wenner, A.M. 1972. Sex ratio as a function of size in marine Crustacea, American Naturalist, Chicago, 106: 321-350.

Williams, S.L. \& M.H. Ruckelshaus. 1993. Effects of nitrogen availability and herbivory on eelgrass (Zostera marina) and epiphytes. Ecology, Durhan, 74 (3): 904-918.

Recebido em 28.IV.2006; aceito em 17.XI.2006.

Revista Brasileira de Zoologia 23 (4): 1207-1216, dezembro 2006 